\title{
Effects of Korean Ginseng and Wild Simulated Cultivation Ginseng for Muscle Strength and Endurance
}

\author{
Eun-Hwa Sohn ${ }^{1 \dagger}$, Yoon Jung Yang ${ }^{2 \dagger}$, Hyun Jung Koo², Dae Won Park ${ }^{2}$, \\ Ye-Jin Kim ${ }^{1}$, Ki Ho Jang ${ }^{3}$, Seung Namkoong ${ }^{4}$ and Se Chan Kang ${ }^{2}$ * \\ ${ }^{1}$ Department of Herbal Medicine Resource, Kangwon National University, Samcheok 245-907, Korea \\ ${ }^{2}$ Department of Life Science, Gachon University, Seongnam 461-701, Korea \\ ${ }^{3}$ Department of Food and Nutrition, Kangwon National University, Samcheok 245-710, Korea \\ ${ }^{4}$ Department of Physical Therapy, Kangwon National University, Samcheok 245-907, Korea
}

\begin{abstract}
Muscle strength and endurance activities of Korean ginseng (Panax ginseng C. A. Meyer; KG) were compared with those of wild simulated cultivation ginseng (WCG) in mice. Fifty male ICR mice were divided into five groups: A (vehicle); B (WCG $100 \mathrm{mg} / \mathrm{kg}$ ); C (WCG $500 \mathrm{mg} / \mathrm{kg}$ ); D (KG $100 \mathrm{mg} / \mathrm{kg}$ ); E (KG $500 \mathrm{mg} / \mathrm{kg}$ ). Subsequently, the mice were subjected to the forced swimming test (FST) and treadmill test at the $4^{\text {th }}$ and $7^{\text {th }}$ weeks. The glycogen content in the muscle and blood analysis (levels of glucose, triglyceride (TG), IGF-1) were also performed immediately after the last FST and treadmill test at the $7^{\text {th }}$ week. Immobility times in FST were shorter in WCG- than KG-treated groups, and the results of the treadmill tests were also significant except for KG-treated at $100 \mathrm{mg} / \mathrm{kg}$. The glycogen content was increased in both groups with a peak at $500 \mathrm{mg} / \mathrm{kg}$ of WCG groups. Serum concentrations of TG and glucose were decreased by administration of KG and WCG and all treated groups showed increase in the level of IGF-1 in serum. These results suggest that KG and WCG supplementations are effective in escalating the muscle strength and endurance.
\end{abstract}

Key words - Wild simulated cultivation ginseng, Muscle strength, IGF-1

\section{Introduction}

It has been reported that many natural resources had an effect on the increase of exercising ability, such as muscle strength and endurance. Among them, especially, studies like the use of ginseng and red ginseng have been carried out (Alvarez et al., 2004; Lee et al., 2009). Korean ginseng (KG), the root of Panax ginseng C.A. Meyer (Araliaceae), is a popular natural health food in Asia. Many studies have been reported that $\mathrm{KG}$ had an effect on neuroprotection (Lee et al., 2011), regulation of body fluid and metabolism (Jia et al., 2010), increase of heart stimulation, anti-diuresis and sexual dysfunction (Becker et al., 1996), improvement of resistance on stress (Qiang et al., 2010), anti-inflammation (Cabral de Oliveira et al., 2001; Park et al., 2010), anti-oxidation (Kim et al., 1996), promotion of immune antibody production (Kim

*Corresponding author. E-mail : sckang73@gachon.ac.kr

${ }^{\dagger}$ Eun-Hwa Sohn and Yoon Jung Yang contributed equally. and Jung, 1987), and anti-cancer (Chang et al., 2002). It has been also known that $\mathrm{KG}$ is closely linked to its protective properties against free radical attack and has nutritional ergogenic aids for enhancing endurance capacity (Chen, 1996; Lee et al., 1999; Maffei Facino et al., 1999; In et al., 2006). KG administration to rats prevented myocardial ischemiareperfusion damage induced by hyperbaric oxygen (Maffei Facino et al., 1999), and ameliorated the muscle injury (Alvarez et al., 2004).

Wild mountain ginseng which are grown naturally in the primitive forest without getting polluted by chemical fertilizer for many years are regarded as one of the most highly medical and preservative valued plants. It is believed that the slowergrowing wild roots, which are harvested at an older age, absorb more curative power from the forest floor (Shin et al., 2001). The method called wild-simulated cultivation can be used to grow ginseng without fungicide sprays and needs expensive establishment costs, and we named this type of ginseng in the study, Wild-simulated Cultivation Ginseng 
(WCG). It has been known that distribution of ginsenoside contents to the WCG and ginseng was similar (Jeong et al., 2010). However, there has been reported that roots of WCG contains ginsenoside $\mathrm{Rg}_{1}$ and $\mathrm{Rb}_{1}$, while ginseng has not.

To our knowledge, the effects of WCG as an ergogenic aid for enhancing muscle endurance capacity has not been clarified yet in animal studies. Here, we investigated the effects on muscle strength and the improvement of endurance through the eccentric exercise and measured glycogen contents in muscle and blood analyses including glucose, triglyceride (TG) and IGF-1 in both WCG and KG supplemented mice.

\section{Materials and Methods}

\section{Preparation of extracts of Korean Ginseng and Wild simulated Cultivation Ginseng}

The Korean ginseng (KG) and Wild simulated Cultivation Ginseng (WCG) were purchased from Jirisan Sansam Farming association corporation (Jeonju-si, Korea, www.jrsansam.com, Cheon-Gyu Ko). The dried roots were grounded and extracted with $70 \%$ ethanol at $70^{\circ} \mathrm{C}$ for 18 hours in a reflux condenser. The boiled extracts were then filtered through Whatman No. 2 filter paper and then concentrated by a vacuum evaporator (Rotary evaporator N-1000, Eyela, Japan) at $40^{\circ} \mathrm{C}$ and then were lyophilized to obtain dried samples.

\section{Experimental Animals}

Male ICR mice $(n=50)$ were purchased from Japan SLC, Inc. at 5 weeks of age, and the five animals were housed together in one cage $(27 \times 17 \times 13 \mathrm{~cm})$ in a controlled environment under a light-dark cycle (lights on at 07:00 and off at 19:00). The experimental procedures were conducted according to the Code of Ethics for Animal Experimentation of Gachon University. After a 1 week acclimation, all mice were randomly divided into five groups (10 mice/group): A (no ginseng intake and saline); B (WCG $100 \mathrm{mg} / \mathrm{kg}$ ); C (WCG 500 mg/kg); D (KG 100 mg/kg); E (KG 500 mg/kg). The solutions were administered as a bolus by gavage, using a curved and ball-tipped intubation needle affixed to a syringe for 7 weeks. All groups were allowed to eat food and drink water freely and the body weights were measured at the $1^{\text {st }}, 4^{\text {th }}$ and $7^{\text {th }}$ weeks. The eccentric exercise was performed on a rodent treadmill and FST test with the modification of following protocol described by Armstrong (Armstrong et al., 1983).

\section{Forced Swimming Test (FST)}

The FST was conducted for endurance enhancement ability. After swimming-acclimation twice a week, the FSTs were carried out on the $4^{\text {th }}$ and $7^{\text {th }}$ week after treatment. Mice were forced to swim in acryl plastic tank $(70 \times 70 \times 60 \mathrm{~cm})$ filled with $23-25^{\circ} \mathrm{C}$ water up to $80 \%$. A mouse was regarded as immobile when it remained still in the water, making only small movements to keep its head above water. The total duration of immobility was measured for 4 minutes after 2 minutes.

\section{Rota Rod Treadmill Test}

The rota rod test was used to assess whether there were gross motor impairments in WCG or KG treated mice. Twentyfour hours before the experiment, all mice were habituated to running on a rota rod treadmill (Rota-rod, Ugobasil) at a speed of $60 \mathrm{rpm}$ until they could remain there for 60 seconds without fail. After the experiment, durations of latency to fall (the running time) were measured.

\section{Glycogen Content in Muscle}

To measure the content of glycogen in skeletal muscle, mice were sacrificed by cervical dislocation and the same amounts of right femoral muscle were collected in all experimental groups. Samples of muscle $(160 \mathrm{mg}$ ) were homogenized in 1 $\mathrm{ml}$ of water at $0^{\circ} \mathrm{C}$. The homogenate was hydrolyzed in $100 \mu \mathrm{l}$ of $\mathrm{HCl} 6 \mathrm{M}$, incubating at $100^{\circ} \mathrm{C}$ for 30 minutes. Subsequently, the samples were cooled, neutralized with $285 \mu \mathrm{l}$ of $\mathrm{KOH} 2 \mathrm{M}$ and centrifuged at $100 \mathrm{~g}$ for 10 minutes. The precipitates were incubated with $0.5 \mathrm{ml}$ of distilled water and $1.0 \mathrm{ml}$ of anthrone (final. $0.02 \%$ ) at $37^{\circ} \mathrm{C}$ for 20 minutes, and were quantified visually at $620 \mathrm{~nm}$ using spectrophotometer.

\section{Biochemical Analyses in Serum}

Blood samples were collected from the infra orbital plexus of each animal and serum was separated by centrifugation. The levels of TG and glucose were measured using a Triglyceride assay kit (BioVision) and Glucose assay kit (Abcam), respec- 
tively. At the end of the experiments, all mice were sacrificed by cervical dislocation and the same amount of left femoral muscle was homogenized in $100 \mathrm{mM}$ of potassium phosphate buffer ( $\mathrm{pH}$ 7.4) to measure enzymatic activity of skeletal muscle. The activity of lactate dehydrogenase (LDH) and ATP content in muscle were measured using QuantiChrom ${ }^{\text {TM }}$ Lactate Dehydrogenase Kit (BioAssay Systems) and ATP colorimetric assay kit (Abcam), respectively. The IGF-1 level in blood was measured using the Quantikine mouse / rat IGF-1 ELISA kit (R\&D Systems).

\section{Statistical Analysis}

Data were presented as means $\pm \mathrm{SD}$. All analyses were performed using SPSS V17.0 (SPSS, Chicago, IL). Probability values of less than 0.05 were significant $\left({ }^{*} p<0.05,{ }^{*} p<\right.$ $0.01)$. Data expressed as mean $\pm \mathrm{SD}$ of three to five independent experiments.

\section{Results}

\section{Effect of KG and WCG on Exercise Tolerance}

To measure the change of endurance, FST was carried out on the $4^{\text {th }}$ and $7^{\text {th }}$ weeks after oral administration. The FST was conducted as a study for evaluating the enhancement of muscle endurance ability. Although there were significant decreased immobility times in WCG and KG groups on the $4^{\text {th }}$ and $7^{\text {th }}$ weeks, KG treated group showed no change in immobility time at a dose of $100 \mathrm{mg} / \mathrm{kg}$ (Fig. 1A). The decreased immobility times means that WCG (100, 500 $\mathrm{mg} / \mathrm{kg}$ )- and $\mathrm{KG}(500 \mathrm{mg} / \mathrm{kg})$-treated groups showed the improvement of exercise tolerance.

\section{Effect of KG and WCG on Myorelaxation}

To confirm the change of exercise tolerance, a treadmill test was performed on the $4^{\text {th }}$ and $7^{\text {th }}$ week after treatment of WCG and KG. Activities on the rotating rod treadmill reflected the levels of myorelaxation. Supplementation of WCG and $\mathrm{KG}$ for 4 and 7 weeks also significantly increased the running time $(p<0.01)$ except for KG-treated at $100 \mathrm{mg} / \mathrm{kg}$. (Fig. 1B). In the treadmill test representing the eccentric exercise, the results of WCG-and KG-treated groups are compatible with those of the FST tests.

\section{Effect of KG and WCG on Glycogen Content in Muscle}

Maintaining adequate stores to meet energy needs help to ameliorate fatigue during exercise. Carbohydrate stores in muscle and liver are important for sustained energy. It has been reported that a low or depleted glycogen stores limit exercise time and intensity and leads to decrease the time to exhaustion during physical activity (Cochran et al., 2010). Muscle glycogen becomes important as a fuel for muscular exercise as the intensity of exercise increases. Compared with the control group, in all WCG- and KG-treated groups, the

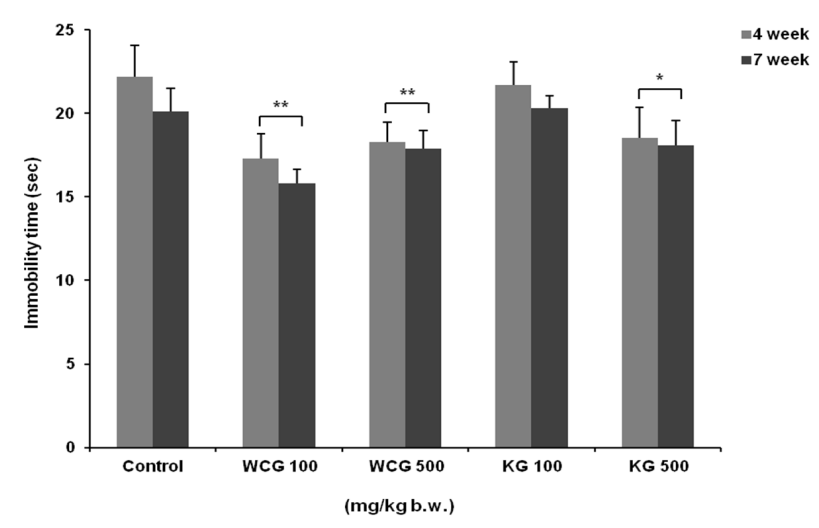

(A)

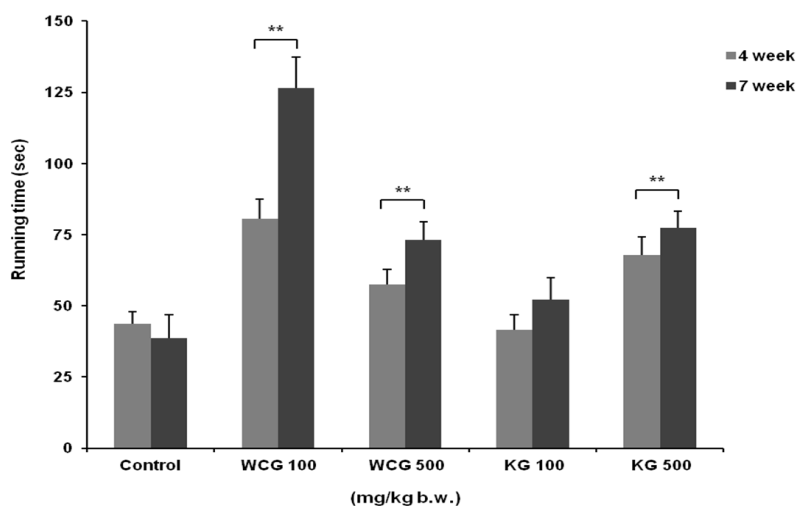

(B)

Fig. 1. (A) Effect of KG and WCG on the force swimming test (FST). After swimming-acclimation twice a week, the FSTs were carried out on the $4^{\text {th }}$ and $7^{\text {th }}$ week after treatment. Mice were dropped into an acryl plastic tank $(70 \times 70 \times 60 \mathrm{~cm})$ filled with $23-25^{\circ} \mathrm{C}$ water up $80 \%$. After 2 minutes, the immobility time was measured for $4 \mathrm{~min}$. (B) Effect of KG and WCG on a rota-rod treadmill test. The running time was measured using treadmills (Rota-rod, Ugobasil) on the $4^{\text {th }}$ and $7^{\text {th }}$ week after treatment of WCG and KG. ** Significantly different from the control at $p<0.01$. 
contents of glycogen in muscle were increased significantly (Fig. 2). The highest level of glycogen in muscle was shown in WCG-treated groups at $500 \mathrm{mg} / \mathrm{kg}$.

\section{Effect of KG and WCG on Levels of Glucose and TG}

In both WCG- and KG-treated groups, the levels of blood glucose were lower than that of the control group (Fig. 3A). There were significant decreases $(p<0.05)$ of blood TG levels in $500 \mathrm{mg} / \mathrm{kg}$ of WCG- and KG-treated group (100 and $500 \mathrm{mg} / \mathrm{kg}$ ) (Fig. 3B), but the levels of TG in muscle did not change in both-treated groups (data not shown).

\section{Effect of KG and WCG on Levels of IGF-1}

IGF-1 plays a crucial role in muscle regeneration and endurance. IGF-1 stimulates both proliferation and differentiation of stem cells in an autocrine-paracrine manner, although it induces differentiation to a much greater degree (Singleton and Feldman, 2001). In all WCG- and KG-treated groups, the levels of IGF-1 in serum were significantly increased $(p<$ 0.05) compared to the control group (Fig. 3C).

\section{Discussion}

WCG and mountain ginseng in the wild are perennial plants belonging to the genus Panax and have been used for

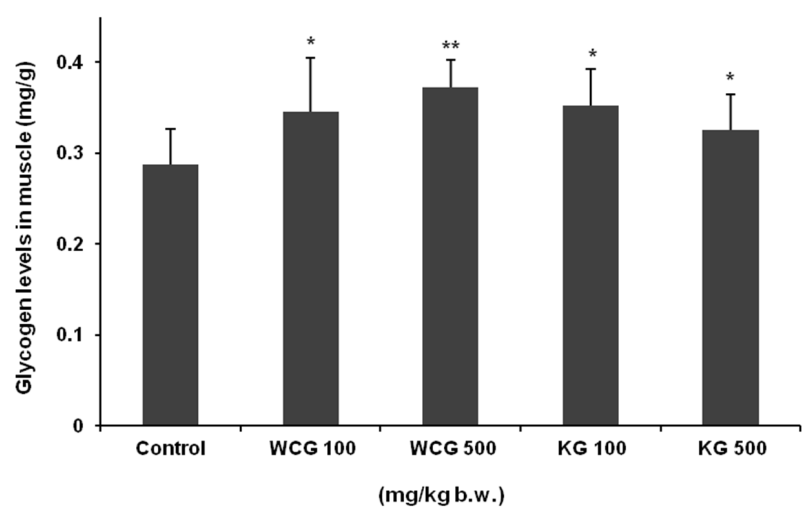

Fig. 2. Effect of KG and WCG on glycogen levels in muscle. Thirty of $\mathrm{KOH}$ solution was added to the skeletal muscles and samples were incubated at $100^{\circ} \mathrm{C}$ for 30 minutes. After incubation, samples were mixed with $95 \%$ of ethanol and centrifuged for 15 minutes at $4,000 \times \mathrm{g}$. The precipitates were incubated with $0.5 \mathrm{ml}$ of distilled water and $1.0 \mathrm{ml}$ of anthrone (final. $0.02 \%$ ) at $37^{\circ} \mathrm{C}$ for 20 minutes, and were quantified visually at $620 \mathrm{~nm}$ using spectrophotometer.

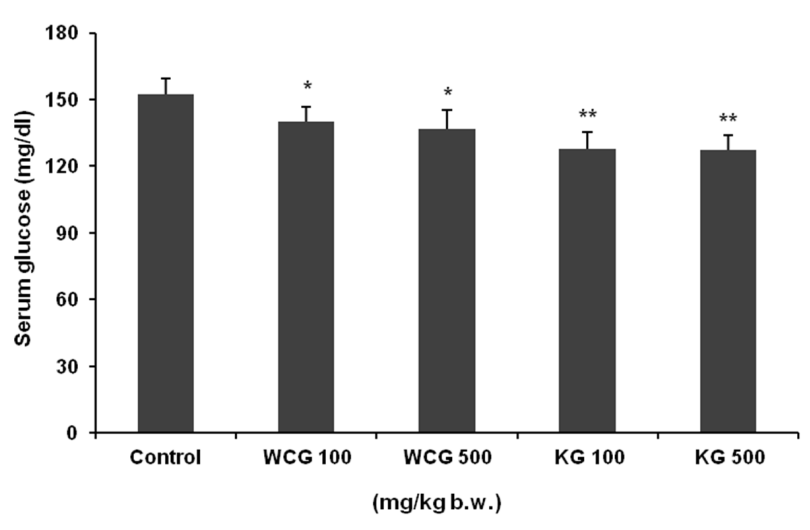

(A)

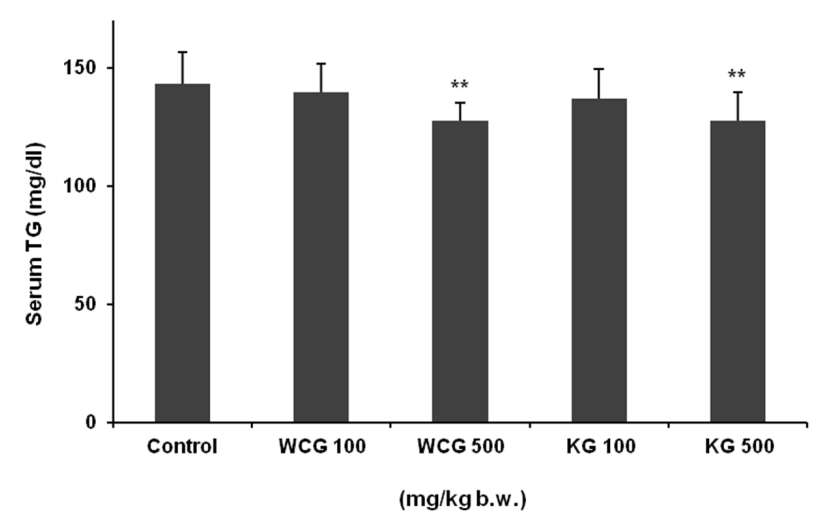

(B)

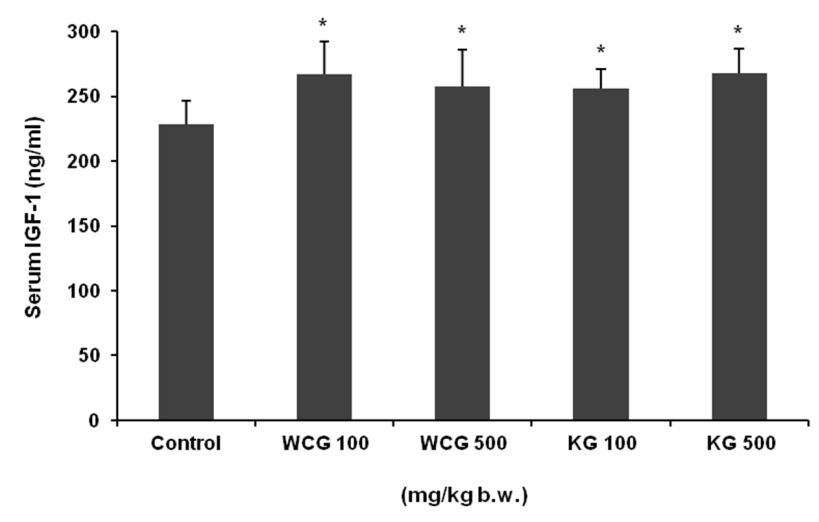

(C)

Fig. 3. (A) Effect of KG and WCG on glucose levels in serum. The levels of glucose were measured using Glucose assay kit. (B) Effect of KG and WCG on TG level in serum. The levels of triglyceride (TG) were measured using Triglyceride assay kit (BioVision). (C) Effect of KG and WCG on IGF-1 concentration in serum. The IGF-1 level in blood was measured using the Quantikine mouse/mouse IGF-1 ELISA kit (R\&D Systems). * Significantly different from the control at $p<0.05$. 
typical energy-supplement herbs in Chinese and oriental medicine. In this study, we investigated the comparative effects of WCG and KG on the strength and the endurance of muscles. WCG-treated groups showed more improved muscle strength and endurance than those of KG-treated groups.

On the $4^{\text {th }}$ and $7^{\text {th }}$ week, all WCG-treated groups and 500 $\mathrm{mg} / \mathrm{kg}$ of the KG-treated group significantly increased the treadmill running time and significantly decreased the immobility time in the FST test. These results suggested that WCGand KG-treated groups showed the improvement of muscle endurance and WCG has a higher effect on the muscle endurance at a lower concentration comparing with those of KG. Generally, the muscle endurance means the recovery of muscle contraction through the inhibition of fatigue materials (e.g. inorganic phosphate and lactic acid in muscle accumulation from glycogen) and the rapid restoration of energy-metabolic materials like ATP and creatine phosphate (Wei et al., 1992).

After the final FST at the $7^{\text {th }}$ week, the contents of glycogen in all WCG- and KG-treated groups were increased significantly compared to the control group. Glycogen is an important energy source during prolonged strenuous exercise and exercise must be abandoned if glycogen stores are depleted. Glycogen storage in the body is limited (300-500 g) (Beelen et al., 2010). As exercise continues, stored glycogen in the skeletal muscle and liver becomes depleted rapidly and exercise performed at $65-75 \%$ of maximal oxygen consumption can be maintained only for 90-120 minutes. Therefore, amelioration of glycogen depletion during prolonged strenuous exercise can be a major determinant of endurance and this accumulation of the glycogen in the muscle by KG and WCG might increase the promotion of endurance and exercise efficiency (Hawley and Burke, 2010). It has been also reported that after exhaustive exercise skeletal muscle glycogen levels of ginseng saponin treated rats were slightly higher than those of saline treated rats (Alvarez et al., 2004). These results suggested the possibility that the lowered consumption of glycogen might be due to the promotion of muscle endurance by WCG and KG supplementations.

Eccentric exercise (EC) produces high muscular tension when the muscles are stretched and is associated to delayed onset muscle soreness provoking ultra-structural and metabolic changes in the muscle cells. The injuries are subcellular and generally in small areas of the muscular fiber. Therefore, they suffer a transient reduction in strength, subsequently muscular pain and finally an inflammation process that causes further tissue deterioration. EC is also responsible for the low levels of glycogen and induces transient insulin resistance in healthy individuals (King et al., 1993; Kirwan et al., 1992).

We also measured the serum analysis such as the levels of TG, glucose and IGF-1 were performed immediately after the last FST and treadmill test at the $7^{\text {th }}$ week. The TG levels in serum were decreased significantly in $500 \mathrm{mg} / \mathrm{kg}$ of WCGand KG-treated groups. However, the TG level in muscle and the activity of LDH and ATP content in muscle were not changed (data not shown). In the glycolysis, $\mathrm{NAD}^{+}$is needed for the ATP production and $\mathrm{NAD}^{+}$supply by $\mathrm{LDH}$ is needed for serial ATP production in anaerobic exercise which resulted in lactic acid formation. These results suggest that WCG and $\mathrm{KG}$ could induce the conversion to aerobic exercise from anaerobic exercise forms the lactic acid. The ATP content in muscle is decreased rapidly in continuous exercise because the energy generated by degradation of ATP is used in the muscle contraction during the exercise. Finally, the glycogen is exhausted and the anaerobic energy metabolism in the muscle is activated which resulted in lactic acid accumulation (Paik et al., 1997). These metabolic materials induce the fatigue after the muscle exercise. With this reason, we analyzed the ATP content in the muscle after WCG and ginseng treatment for weeks, but WCG- and KG-treated mice did not show any changes that the ATP content in the muscle (data not shown). Therefore, these results suggest that WCG and KG were used for the ATP effectively to inhibit the rapid decrease of ATP. Overall glucose levels in serum showed the low pattern in all WCG- and KG-treated groups. These results suggest that WCG and KG might enhance the consumption of glucose in serum during FST and treadmill tests.

IGF-1 is the polypeptide consisting of 70 kinds of amino acids and is produced mainly in the liver and bone. It has been reported that IGF-1 stimulated the protein synthesis, cell differentiation, growth and regeneration of skeletal muscle and protected the neuronal cell (Singleton and Feldman, 2001). Also, it has been reported that the declines of the muscle strength and exercise ability were closely related with the decrease of IGF-1 concentration in the body and the 
absence of IGF-1 causes the lack of growth, the retardation of spiritual development and the insensibility of sensory nerves (Laron, 2001; Nilsson et al., 1986). In this study, the levels of blood IGF-1 were significantly increased in all treated groups and these results suggested that WCG and KG stimulated the synthesis of IGF-1 resulting to the alleviation of stamina reduction and the improvement of the muscle endurance. The beneficial effect of WCG can be attributed to the activity of ginsenoside $\mathrm{Rg} 1$ and $\mathrm{Rb}_{1}$.

Due to the relatively high cost of WCG used in this study, we were unable to examine with greater parameters. Additional analyses are required to detect a clear statistical difference regarding the parameters between WCG and KG. In conclusion, we verified that WCG and KG had effects on the muscle strength and improvement of endurance.

\section{Acknowledgement}

This work was supported by the Gachon University research fund of 2012.

\section{Literature Cited}

Alvarez, A.I., A.C. Cabral de Oliveira, A.C. Perez, L. Vila, A. Ferrando and J.G. Prieto. 2004. The effect of ginseng on muscle injury and inflammation. J. Ginseng Res. 28:18-26.

Armstrong, R.B., R.W. Ogilvie and J.A. Schwane. 1983. Eccentric exercise-induced injury to rat skeletal muscle. J. Appl. Physiol. 54:80-93.

Becker, B.N., J. Greene, J. Evanson, G. Chidsey and W.J. Stone. 1996. Ginseng-induced diuretic resistance. J. Am. Med. Assoc. 276:606-607.

Beelen, M., L.M. Burke, M.J. Gibala and L. JC. van Loon. 2010. Nutritional strategies to promote postexercise recovery. Int. J. Sport Nutr. Exerc. Metab. 20:515-532.

Cabral de Oliveira, A.C., A.C. Perez, G. Merino, J.G. Prietp and A.I. Alvarez. 2001. Protective effects of Panax ginseng on muscle injury and inflammation after eccentric exercise. Comp. Biochem. Physiol. C Toxicol. Pharmacol. 130:369-377. Chang, T.K., J. Chen and S.A. Benetton. 2002. In vitro effect of standardized ginseng extracts and individual ginsenosides on the catalytic activity of human CYP1A1, CYP1A2, and CYP1B1. Drug Metab. Dispos. 30:378-384.
Chen, X. 1996. Cardiovascular protection by ginsenosides and their nitric oxide releasing action. Clin. Exp. Pharmacol. Physiol. 23:728-732.

Cochran, A.J., J.P. Little, M.A. Tarnopolsky and M.J. Gibala. 2010. Carbohydrate feeding during recovery alters the skeletal muscle metabolic response to repeated sessions of highintensity interval exercise in humans. J. Appl. Physiol. 108: 628-636.

Hawley, J.A. and L.M. Burke. 2010. Carbohydrate availability and training adaptation: effects on cell metabolism. Exerc. Sport Sci. Rev. 38:152-160.

In, J.G., B.S. Lee, E.J. Kim, M.H. Park and D.C. Yang. 2006. Increase of functional saponin by acidic treatment and temperature of red ginseng extract. Korean J. Plant Res. 19:139-143.

Jeong, H.S., C.S. Lim, B.C. Cha, S.H. Choi and K.R. Kwon. 2010. Component analysis of cultivated ginseng, cultivated wild ginseng, and wild ginseng and the change of ginsenoside components in the process of red ginseng. J. Pharmacopuncture 13:63-77.

Jia, Y., Z.Y. Li. H.G. Zhang, H.B. Li, Y. Liu and X.H. Li. 2010. Panax notoginseng saponins decrease cholesterol ester via up-regulating ATP-binding cassette transporter A1 in foam cells. J. Ethnopharmacol. 132:297-302.

Kim, J.S., K.W. Kim, K.J. Choi, Y.K. Kwak, K.S. Im, K.M. Lee and H.Y. Chung. 1996. Screening of anti-oxidative components from red ginseng saponin. Korean J. Ginseng Sci. 20:173-178.

Kim, M.J. and N.P. Jung. 1987. The effects of ginseng saponin on the mouse immune system. Korean J. Ginseng Sci. 11: 130-135.

King, D.S., T.L. Feltmeyer, P.J. Baldus, R.L. Sharp and J. Nespor. 1993. Effects of eccentric exercise on insulin secretion and action in humans. J. Appl. Physiol. 75:2151-2156.

Kirwan, J.P., R.C. Hickner, K.E. Yarasheski, W.M. Kohrt, B.V. Wiethop and J.O. Holloszy. 1992. Eccentric exercise induces transient insulin resistance in healthy individuals. J. Appl. Physiol. 72:2197-2202.

Laron, Z. 2001. Insulin-like growth factor 1 (IGF-1): a growth hormone. Mol. Pathol. 54:311-316.

Lee, B.J., J.W. Kim, E.Y. Ji, S.Y. Yun, S.M. Lee and J.H. Lew. 2009. Anxiolytic and antidepressive effect of non-saponin fraction of Korean red ginseng. Kor. J. Herbology 24:143-148.

Lee, H.J., D.Y. Kim and C.C. Chang. 1999. Antioxidant effects of Korean red ginseng components on the antioxidant enzymes activity and liver peroxidation in the liver of mouse treated 
with paraquat. J. Ginseng Res. 23:182-189.

Lee, J.S., H.S. Choi, S.W. Kang, J.H. Chung, H.K. Park, J.Y. Ban, O.Y. Kwon, H.P. Hong and Y.G. Ko. 2011. Therapeutic effect of Korean red ginseng on inflammatory cytokines in rats with focal cerebral ischemia/reperfusion injury. Am. J. Chin. Med. 39:83-94.

Maffei Facino, R., M. Carini, G. Aldini, F. Berti and G. Rossoni. 1999. Panax ginseng administration in the rat prevents myocardial ischemia-reperfusion damage induced by hyperbaric oxygen: evidence for an antioxidant intervention. Planta. Med. 65:614-619.

Nilsson, A., J. Isgaard, A. Lindahl, A. Dahlström, A. Skottner and O.G. Isaksson. 1986. Regulation by growth hormone of number of chondrocytes containing IGF-I in rat growth plate. Science 233:571-574.

Paik, I.Y., J.K. Kim, Y.S. Chun and H.J. Oho. 1997. Verifying the validity of fatigue elements changes following absolute exercise intensities. Kor. J. Physical. Edu. 36:218-223.

Park, H.J., D.H. Jung, H. Joo, N.S. Kang, S.A. Jang, J.G. Lee and E.H. Sohn. 2010. The comparative study of anti-allergic and anti-inflammatory effects by fermented red ginseng and red ginseng. Korean J. Plant Res. 23(5):415-422.

Qiang, H., C. Zhang, Z.B. Shi, H.Q. Yang and K.Z. Wang. 2010. Protective effects and mechanism of Panax Notoginseng saponins on oxidative stress-induced damage and apoptosis of rabbit bone marrow stromal cells. Chin. J. Integr. Med. 16:525-530.

Shin, S.S., K.C. Kim, Y.H. Choi, Y.T. Lee, H.S. Eom and C.S. Kim. 2001. Critic standardization and objectivity of mountain grown ginseng. J. Dong-Eui Oriental Med. 5:107-114 (in Korean).

Singleton, J.R. and E.L. Feldman. 2001. Insulin-like growth factor-I in muscle metabolism and myotherapies. Neurobiol. Dis. 8:541-554.

Wei, R., C. Chen, J. Dong, S. Qiu and Z. Cao. 1992. Effect of radix Astragali and radix ginseng in enhancing the metabolism of human myocardial cells in vitro. Zhongguo. Zhong. Yao. Za. Zhi. 17:173-175.

(Received 15 October 2012 ; Revised 23 November 2012 ; Accepted 3 December 2012) 